\title{
MEAN GROWTH OF BLOCH FUNCTIONS AND MAKAROV'S LAW OF THE ITERATED LOGARITHM
}

\author{
RODRIGO BAÑUELOS AND CHARLES N. MOORE
}

(Communicated by J. Marshall Ash)

\begin{abstract}
The authors construct an example of a Bloch function on the unit disc whose circular $L^{2}$ means grow at the maximal possible rate but which has no lower bound in the law of the iterated logarithm for Bloch functions. This answers a question of Przytycki [4, p. 154] and Makarov [3, p. 42].
\end{abstract}

\section{INTRODUCTION}

Let $f$ be holomorphic on the unit disc $D$, and suppose $f \in \mathscr{B}$ (the Bloch class), that is, $\|f\|_{\mathscr{B}}=|f(0)|+\sup _{z \in D}(1-|z|)\left|f^{\prime}(z)\right|<\infty$. The law of the iterated logarithm (LIL) of Makarov [2] states that

$$
\limsup _{\rho \rightarrow 1} \frac{|f(\rho z)|}{\sqrt{\log \frac{1}{1-\rho} \log \log \log \frac{1}{1-\rho}}} \leq C\|f\|_{\mathscr{B}}
$$

for almost all $z \in \partial D$ where $C$ is a constant independent of $f$ or $z$.

The simplest examples of functions in $\mathscr{B}$ are provided by lacunary series of the form $f(z)=\sum_{n=1}^{\infty} a_{n} z^{q^{n}}$ where $\left\{a_{n}\right\} \in l^{\infty}$ and $q$ is a positive integer. For $f \in \mathscr{B}$ we set $B_{\rho}=\int_{\partial D}|f(\rho z)|^{2}|d z| / 2 \pi$. Suppose $f$ is a lacunary series in $\mathscr{B}$ which has $B_{\rho} \rightarrow \infty$ as $\rho \rightarrow 1$. Then the classical LIL for lacunary series (see Salem-Zygmund [5], Erdös-Gál [1], or M. Weiss [7]) states that $\lim \sup _{\rho \rightarrow 1}|f(\rho z)| / \sqrt{B_{\rho} \log \log B_{\rho}}=1$ for almost all $z \in \partial D$. Thus, for such an $f$ we conclude: If

$$
\limsup _{\rho \rightarrow 1} \frac{B_{\rho}}{\log \frac{1}{1-\rho}}=0
$$

then

$$
\limsup _{\rho \rightarrow 1} \frac{|f(\rho z)|}{\sqrt{\log \frac{1}{1-\rho} \log \log \log \frac{1}{1-\rho}}}=0
$$

Received by the editors January 25, 1990 and, in revised form, June 13, 1990.

1980 Mathematics Subject Classification (1985 Revision). Primary 30B30.

The first author was supported in part by the NSF.

The second author was supported by an NSF Postdoctoral Fellowship. 
a.e. on $\partial D$ and, if

$$
\limsup _{\rho \rightarrow 1} \frac{|f(\rho z)|}{\sqrt{\log \frac{1}{1-\rho} \log \log \log \frac{1}{1-\rho}}}=0
$$

on a set of positive measure in $\partial D$, then

$$
\liminf _{\rho \rightarrow 1} \frac{B_{\rho}}{\log \frac{1}{1-\rho}}=0 .
$$

In fact, (1.1) and (1.2) are true for all lacunary series in $\mathscr{B}$ since they are also trivially true if $B_{\rho}$ remains bounded as $\rho \rightarrow 1$. Thus, it is natural to conjecture (Przytycki [4] or Makarov [3]) that (1.1) and (1.2) remain valid for all $f \in \mathscr{B}$. D. Ullrich [6] has constructed an example of an $f \in \mathscr{B}$ for which (1.1) fails. The purpose of this note is to construct an $f \in \mathscr{B}$ for which (1.2) fails.

We also remark that Przytycki [4] conjectured a variant of (1.2): If

$$
\limsup _{\rho \rightarrow 1} \frac{|f(\rho z)|}{\sqrt{\log \frac{1}{1-\rho} \log \log \log \frac{1}{1-\rho}}}=0,
$$

on a set of positive measure in $\partial D$, then

$$
\limsup _{\rho \rightarrow 1} \frac{B_{\rho}}{\log \frac{1}{1-\rho}}=0 .
$$

This is false even for lacunary series; an example is provided by the function $f(z)=\sum_{n=1}^{\infty} \sum_{j=0}^{\mathscr{B}(n)} z^{2^{(\mathscr{B}(n)+j)}}$ where $\mathscr{B}(n)=2^{2^{n}}$ (see also Makarov [3]).

\section{Construction}

We will construct a function $f$ of the form $f(z)=\sum_{n=4}^{\infty} a_{n} z^{n}=\sum_{k=1}^{\infty} b_{k}(z)$ where $b_{k}(z)=a_{4^{k}} z^{4^{k}}+a_{4^{k}+1} z^{4^{k}+1}+\cdots+a_{4^{k+1}-1} z^{4^{k+1}-1}$ which satisfies:

(a) $\left\|b_{k}\right\|_{\infty} \leq 1$ for every $k$,

(b) $\liminf _{n \rightarrow \infty} \sum_{j=4}^{n} a_{j}^{2} / \log n>0$, and

(c) $\lim \sup _{n \rightarrow \infty}\left|\sum_{k=1}^{n} b_{k}(z)\right| / \sqrt{n \log \log n}=0$ for all $z \in \partial D$.

It is well known [2, 4], that (a) implies $f \in \mathscr{B}$ and that the quantities in (b) and (c) for the partial sums of $f$ are comparable to the corresponding quantities for the Abel means of $f$ in conjecture (1.2), so that it suffices to construct $f$ having (a), (b), and (c). To further simplify the construction, we will momentarily show that it will be sufficient to construct $f(z)$ which has:

(i) $\left\|b_{j}\right\|_{\infty} \leq 1$ for all $j$.

(ii) If $4^{l} \leq j<4^{l+1}$ then $\left|\sum_{i=4^{\prime}}^{j} b_{i}\right| \leq \sqrt{4^{l+1}}$.

(iii) If $4^{l} \leq j<4^{l+1}$ then $\left\|\sum_{i=4^{l}}^{j} b_{i}\right\|_{2}^{2} \geq \frac{2}{5}\left(j+1-4^{l}\right)$. 
Clearly, (i) implies (a). Now fix $n$ and choose $m$ so that $4^{m-1} \leq n<4^{m}$. Then

$$
\sum_{j=1}^{n} a_{j}^{2} \geq \sum_{j=1}^{4^{m-1}-1} a_{j}^{2}=\left\|\sum_{i=1}^{m-2} b_{i}\right\|_{2}^{2} \geq \frac{2}{5}(m-2) \geq C \log n,
$$

where we have made repeated use of (iii) for the next to the last inequality. Thus, (iii) implies (b). Fix $n$ again and pick $l$ so that $4^{l} \leq n<4^{l+1}$. Then by repeated use of (ii) we have

$$
\left|\sum_{i=1}^{n} b_{i}\right| \leq\left|\sum_{i=4^{l}}^{n} b_{i}\right|+\sum_{j=1}^{l}\left|\sum_{i=4^{l-j}}^{4^{-j+1}-1} b_{i}\right| \leq \sum_{i=1}^{l+1} \sqrt{4^{j}} \leq C \sqrt{4^{l}}<C \sqrt{n}
$$

so that (c) trivially follows from (ii).

Our inspiration for the construction of $f$ having (i)-(iii) comes from the construction of a dyadic martingale satisfying these properties. We will inductively define the $b_{k}$ in such a way so that the partial sums of the $b_{k}$ are "stopped" at the places where they begin to grow too large, and thus we will get an estimate like (ii). However, we must continue to add enough so that (iii) holds.

To construct our $f$, it suffices to fix a nonnegative integer $l$ and construct the functions $b_{j}, j=4^{l}, 4^{l}+1, \ldots, 4^{l+1}-1$ having (i)-(iii). For $j=4^{l}$ we set $b_{j}=z^{4^{j}}$ and for $j=4^{l}+1,4^{l}+2, \ldots, 4^{l+1}-1$ we recursively define

$$
b_{j}(z)=\left(1-4^{-(l+1)}\left|\sum_{i=4^{l}}^{j-1} b_{i}(z)\right|^{2}\right) z^{2 \cdot 4^{j}} \text {. }
$$

To see that this works, we first note that (i)-(iii) hold for $b_{j}(z)$ if $j=4^{l}$. We now fix $k$ such that $4^{l}<k \leq 4^{l+1}-1$ and assume that (i)-(iii) hold for all $j=4^{l}, 4^{l}+1, \ldots, k-1$. We also assume that for each such $j$ the function $b_{j}(z)$ is of the proper form, that is, $\hat{b}_{j}(n)=0$ if $n \notin\left\{4^{j}, 4^{j}+1, \ldots, 4^{j+1}-1\right\}$, where $\hat{b}_{j}(n)$ represents the coefficient of $z^{n}$ in the series expansion of $b_{j}(z)$. First note that if we set $g(z)=1-4^{-(l+1)}\left|\sum_{i=4^{l}}^{k-1} b_{i}(z)\right|^{2}$ then $\hat{g}(n)=0$ if $n \notin\left\{-4^{k},-4^{k}+1, \ldots, 0, \ldots, 4^{k}-1,4^{k}\right\}$. So if $n<2 \cdot 4^{k}-4^{k}=4^{k}$ then $\hat{b}_{k}(n)=0$. Also, if $n>4^{k}+2 \cdot 4^{k}=3 \cdot 4^{k}$ then $\hat{b}_{k}(n)=0$. But $3 \cdot 4^{k}<4^{k+1}-1$ so we conclude that $\hat{b}_{k}(n)=0$ if $n \notin\left\{4^{k}, \ldots, 4^{k+1}-1\right\}$ and thus $b_{k}(z)$ has the desired form.

Now note that by the induction hypothesis, $\left|\sum_{i=4^{l}}^{k-1} b_{i}(z)\right|^{2} \leq 4^{l+1}$ so that trivially we have $\left\|b_{k}\right\|_{\infty} \leq 1$. Also note that

$$
\left|\sum_{i=4^{l}}^{k} b_{i}(z)\right| \leq\left|\sum_{i=4^{l}}^{k-1} b_{i}(z)\right|+\left|b_{k}(z)\right| \leq\left|\sum_{i=4^{l}}^{k-1} b_{i}(z)\right|+1-4^{-(l+1)}\left|\sum_{i=4^{l}}^{k-1} b_{i}(z)\right|^{2} \leq \sqrt{4^{l+1}} \text {. }
$$


Here we have used the fact that if $l \geq 0$ and if $x$ is a number such that $0 \leq x \leq \sqrt{4^{l+1}}$ then $x+1-4^{-(l+1)} x^{2} \leq \sqrt{4^{l+1}}$. (This is an easy calculcus exercise.) Thus (ii) holds for $j=k$.

Furthermore,

$$
\begin{aligned}
\left\|\sum_{i=4^{l}}^{k} b_{i}(z)\right\|_{2}^{2} & =\left\|\sum_{i=4^{l}}^{k-1} b_{i}(z)\right\|_{2}^{2}+\left\|b_{k}(z)\right\|_{2}^{2} \\
& \geq\left\|\sum_{i=4^{l}}^{k-1} b_{i}(z)\right\|_{2}^{2}+1-2 \cdot 4^{-(l+1)}\left\|\sum_{i=4^{l}}^{k-1} b_{i}(z)\right\|_{2}^{2} \\
& \geq \frac{2}{5}\left(k-4^{l}\right)\left(1-2 \cdot 4^{-(l+1)}\right)+1 \\
& =\frac{2}{5}\left(k-4^{l}-2 \cdot 4^{-(l+1)} k+\frac{1}{2}\right)+1 \\
& \geq \frac{2}{5}\left(k-4^{l}-\frac{3}{2}\right)+1 \\
& =\frac{2}{5}\left(k+1-4^{l}\right)
\end{aligned}
$$

so (iii) holds for $j=k$ and we are done.

\section{REFERENCES}

1. P. Erdös and I. S. Gál, On the law of the iterated logarithm, Nederl. Akad. Wetensch. Proc. Ser. A 58 (1955), 65-84.

2. N. G. Makarov, On the distortion of boundary sets under conformal mappings, Proc. London Math. Soc. 51 (1985), 369-384.

3. __ Probability methods in conformal mappings I, II, LOMI preprints, USSR Acad. Sci. Steklov Math. Inst. Leningrad, 1988.

4. F. Przytycki, On the law of the iterated logarithm for Bloch functions, Studia Math. 93 (1989), 145-154.

5. R. Salem and A. Zygmund, La loi du logarithme itéré pour les séries trigonométriques lacunaires, Bull. Sci. Math. 74 (1950), 209-224.

6. D. Ullrich, personal communication.

7. M. Weiss, The law of the iterated logarithm for lacunary trigonometric series, Trans. Amer. Math. Soc. 91 (1959), 444-469.

Department of Mathematics, Purdue University, West Lafayette, Indiana 47907

Department of Mathematics, Washington University, St. Louis, Missouri 63130

Current address: Department of Mathematics, Kansas State University, Manhattan, Kansas 66506 